\title{
Self-Assembled Nanofeatures in Complex Three-Dimensional Topographies via Nanoimprint and Block Copolymer Lithography Methods
}

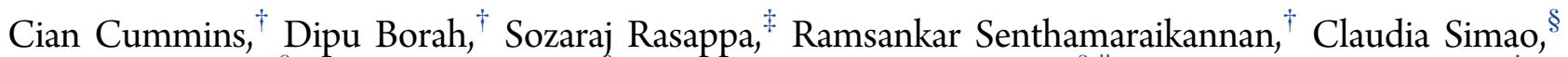 \\ Achille Francone, ${ }^{\S}$ Nikolaos Kehagias, ${ }^{\S}$ Clivia M. Sotomayor-Torres, ${ }^{\S, \|}$ and Michael A. Morris, ${ }^{*}{ }^{\dagger}$ \\ ${ }^{\dagger}$ AMBER Centre and CRANN, Trinity College Dublin, Dublin 2, Ireland \\ ${ }^{\ddagger}$ Optoelectronics Research Center, Tampere University of Technology, P.O. Box 692, FI-33101 Tampere, Finland \\ ${ }^{\S}$ Catalan Institute of Nanoscience and Nanotechnology (ICN2), CSIC and The Barcelona Institute of Science and Technology, \\ Campus UAB, Bellaterra, 08193 Barcelona, Spain \\ "ICREA, Pg. Lluís Companys 23, 08010 Barcelona, Spain
}

Supporting Information

\begin{abstract}
Achieving ultrasmall dimensions of materials and retaining high throughput are critical fabrication considerations for nanotechnology use. This article demonstrates an integrated approach for developing isolated sub-20 (ii) (ii) (iii) $\mathrm{nm}$ silicon oxide features through combined "top-down" and "bottom-up" methods: nanoimprint lithography (NIL) and block copolymer (BCP) lithography. Although techniques like those demonstrated here have been developed for nanolithographic application in the microelectronics processing industry, similar approaches could be utilized for sensor, fluidic, and optical-based devices. Thus, this article centers on looking at the possibility of generating isolated silica structures on substrates. NIL was used to create intriguing three-dimensional (3-D) polyhedral oligomeric silsesquioxane (POSS) topographical arrays that guided and confined polystyrene-block-poly(dimethylsiloxane) (PS$b$-PDMS) BCP nanofeatures in isolated regions. A cylinder forming PS- $b$-PDMS BCP system was successfully etched using a one-step etching process to create line-space arrays with a period of $35 \mathrm{~nm}$ in confined POSS arrays. We highlight large-area (>6 $\mu \mathrm{m})$ coverage of line-space arrays in 3-D topographies that could potentially be utilized, for example, in nanofluidic systems. Aligned features for directed self-assembly application are also demonstrated. The high-density, confined silicon oxide nanofeatures in soft lithographic templates over macroscopic areas illustrate the advantages of integrating distinct lithographic methods for attaining discrete features in the deep nanoscale regime.
\end{abstract}

\section{INTRODUCTION}

Scientific interest in developing innovative patterning methods based on "bottom-up" or "top-down" paradigms that allow fabrication of sub-100 $\mathrm{nm}$ features for semiconductor feature size scaling has increased immensely over the past decade. ${ }^{1,2}$ The ability to generate ultrahigh-density and highly resolved patterns using conventional UV photolithography at appropriate costs has necessitated the search for a nontraditional patterning technique to augment the lithographic process. Electron beam lithography (EBL) can possibly achieve the desired dimensions; however, its serial write scheme is a major drawback for photomask development. ${ }^{3}$ Nanoimprint lithography (NIL) has emerged as a promising technique for the robust formation of sub-50 nm features. In principle, NIL is a straightforward concept based on a mechanical force being applied on a mold (that contains features to be imprinted) atop a thermal- or ultraviolet-sensitive resist layer. ${ }^{4}$ Research on NIL materials (both molds and resists) to enhance the final pattern integrity has advanced endlessly. ${ }^{5}$ In parallel, industry-driven efforts are heavily investigating directed self-assembly (DSA) of block copolymers (BCPs) to reach ultrasmall features for increased device performance. ${ }^{6,7}$ However, in addition to this obvious application area, integrating scalable patterning processes with high throughput could be significant to meeting other nanotechnological needs and may be applied in areas where the placement of features is not as stringent as integrated circuit layouts, for example, in sensors, membranes, and batteries. $^{8,9}$

To date, DSA demonstration has primarily used either topographical (graphoepitaxy) or chemical (chemoepitaxy) prepatterns to guide BCP features. ${ }^{10-20}$ More recently, synergistic approaches that combine NIL and BCP lithography have shown significant promise for DSA. Here, the guiding topography is created using reusable NIL stamps to replicate features. For example, Olynick and co-workers ${ }^{21}$ used a silicon mold coated with a poly(dimethylsiloxane) (PDMS) brush layer to imprint a polystyrene (PS)-block-PDMS (PS-b-PDMS) $\mathrm{BCP}$ thin film to fabricate sub-10 $\mathrm{nm}$ features. It was found that

Received: June 13, 2017

Accepted: July 18, 2017

Published: August 10, 2017 
Scheme 1. Process Integrating NIL and BCP Lithography To Achieve Silicon Oxide Nanofeatures

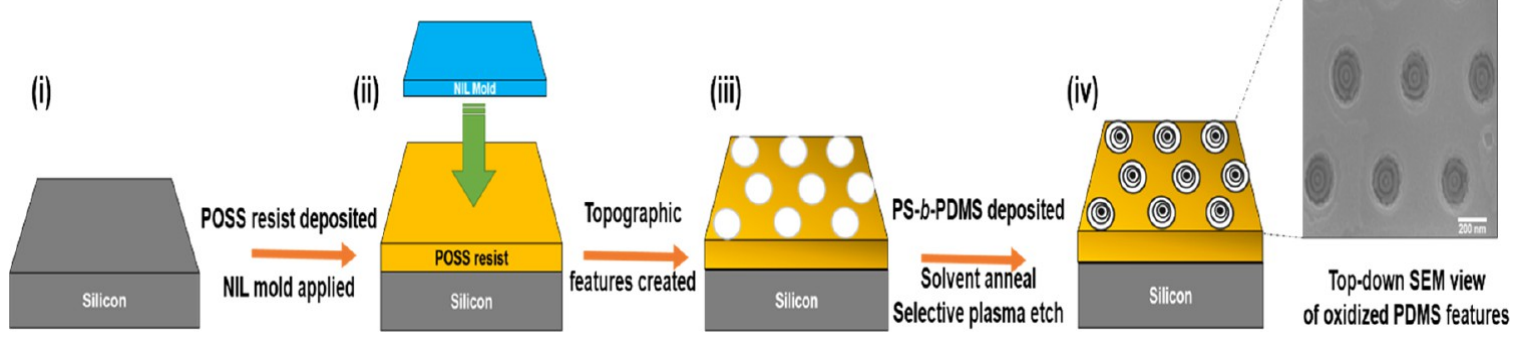

the mold coated with a PDMS brush layer was easily unmolded from the PS- $b$-PDMS films. Molds were used up to 10 times before reapplication of the PDMS brush layer was necessary. Additionally, Seagate has reported excellent demonstrations of sphere-forming PS- $b$-PDMS systems (at $>1$ teradot/in. ${ }^{2}$ ) with NIL (and optical lithography) for application in developing ultrahigh-density bit-patterned magnetic storage media.,22,23 We have previously utilized NIL and BCP methods for DSA purposes on polyhedral oligomeric silsesquioxane (POSS)derived substrates to create aligned line-space and pillar features with excellent coverage over large areas. ${ }^{24}$ Resist designs have been optimized to show control over cylinder orientation depending on the existence or absence of trench base material.

The key objective of this current work is to expand NIL and BCP lithography to nontraditional guiding templates, which may open up large-area-controlled routes to patterning of nanometer features for alternative nanoapplications in biomolecule detection, host arrays for complex material deposition, and scaffold materials for filtration media. Although ease of fabrication is attractive for such applications, the ability to reproducibly create large surface coverage of nanofeatures is advantageous. Little work has been carried out on examining the self-assembly of BCPs in nontraditional guiding templates. In this work, soft graphoepitaxy is employed via the imprinting of a PDMS mold on a POSS resist to confine BCP material following solvent vapor annealing (SVA). Organized PS- $b$ PDMS BCP films were etched to produce uniform high-density silicon dioxide line-space features confined within the threedimensional (3-D) NIL POSS resist topographies. The results described in this report extend the level of sophistication of NIL/BCP templating and, in particular, for achieving highthroughput guiding patterns over large areas using NIL, surpassing the often used EBL processes that are expensive and time consuming.

\section{EXPERIMENTAL SECTION}

2.1. UV-NIL Imprinting on Epoxy-POSS Resin. POSS geometries on silicon substrates were fabricated using UV-NIL with elastomeric molds (so-called "soft UV-NIL") in the POSS resist. Epoxy-POSS resin used in the present investigation was generously provided by PROFACTOR $\mathrm{GmbH}$, Austria. The silicon masters were prepared using EBL. Prior to imprinting, original silicon oxide master molds were vapor-coated with an antisticking layer of $1 \mathrm{H}, 1 \mathrm{H}, 2 \mathrm{H}, 2 \mathrm{H}$-perfluorodecyltrichlorosilane (Optool DSX; Daikin Chemical). This allows easy mold release after the imprinting and curing processes. The antisticking layer had a thickness of about $3 \mathrm{~nm}$ and resulted in a surface free energy of $110.5 \mathrm{mN} / \mathrm{m}$ for even demolding. PDMS stamps were fabricated using the original silicon oxide master molds.
Resin solutions were made by dissolving appropriate amounts of the POSS resist in propylene glycol monomethyl ether acetate, followed by the addition of $2 \mathrm{~mol} \%$ (relative to epoxy groups) Irgacure 250 (photoinitiator absorbing in the wavelength range of $275-325 \mathrm{~nm}$ ) and $0.5 \mathrm{wt} \%$ Genocure ITX (sensitizer absorbing in the wavelength range of 340-400 nm) to provide UV sensitivity. Solutions were spin-coated on silicon substrates previously surface-treated with $\mathrm{O}_{2}$ plasma (to remove contamination) to form resist films of various thicknesses.

The imprinting process was performed immediately by repeatedly stamping the PDMS molds over the resin-coated substrates at a pressure of $200 \mathrm{kPa}$ and exposing to UV radiation (365 $\mathrm{nm}$ in wavelength) for $3 \mathrm{~min}$ to cure the resist. The process formed a resist residual layer $(\approx 15 \mathrm{~nm}$ thick $)$ at the bottom of the topography that was removed by $\mathrm{CF}_{4}(15$ $\mathrm{sccm}$ ) plasma treatment for $5 \mathrm{~s}$ with inductively coupled plasma (ICP) and reactive ion-etching (RIE) powers of 400 and $30 \mathrm{~W}$, respectively, at $2.0 \mathrm{~Pa}$ with a helium backside cooling pressure of $1333.2 \mathrm{~Pa}$.

2.2. Deposition of PS-b-PDMS and Solvent Vapor Annealing. Thin films of PS-b-PDMS (31-11k, polydispersity = 1.12; purchased from Polymer Source Inc., Canada) were prepared by depositing a dilute solution (1.0 wt \%) of BCP in toluene onto POSS substrates. As-cast thin films were solventannealed in glass jars under saturated toluene environment at room temperature $(\approx 288 \mathrm{~K})$ for $3 \mathrm{~h}$. Samples were removed from the glass jars after the desired anneal time and allowed to evaporate the trapped solvent under ambient conditions.

2.3. Plasma Etching of PS- $b$-PDMS Films to Form Silicon Oxide Features. Solvent-annealed BCP films were subjected to an initial $\mathrm{SF}_{6}$ plasma $(7 \mathrm{sccm})$ with $30 \mathrm{~W}$ RIE and $50 \mathrm{~W}$ ICP powers at $2.0 \mathrm{~Pa}$ pressure for $3 \mathrm{~s}$ to remove the upper PDMS wetting layer. This was followed by an $\mathrm{O}_{2}$ plasma treatment for $10 \mathrm{~s}$ with $2000 \mathrm{~W}$ ICP and $400 \mathrm{~W}$ RIE powers at 2.0 Pa pressure to selectively etch the exposed PS component. These steps followed a similar methodology developed by Ross and Jung. ${ }^{25}$

2.4. Contact Angle and Surface Free Energy. Contact angles were measured by the static sessile drop method. Surface free energy was calculated from the measured contact angles of deionized water, diiodomethane, and ethylene glycol using the Owens-Wendt model. ${ }^{26}$ Contact angles and surface free energy measurements were carried out using a Krüss DSA 100 goniometer.

2.5. Scanning Electron Microscopy (SEM). SEM images of etched BCP films were obtained by a Field Emission Zeiss Ultra Plus SEM with a Gemini column operating at an accelerating voltage of $3 \mathrm{kV}$. All SEM images shown are following PS removal by $\mathrm{O}_{2}$ plasma leading to consequential oxidation of the PDMS block. 

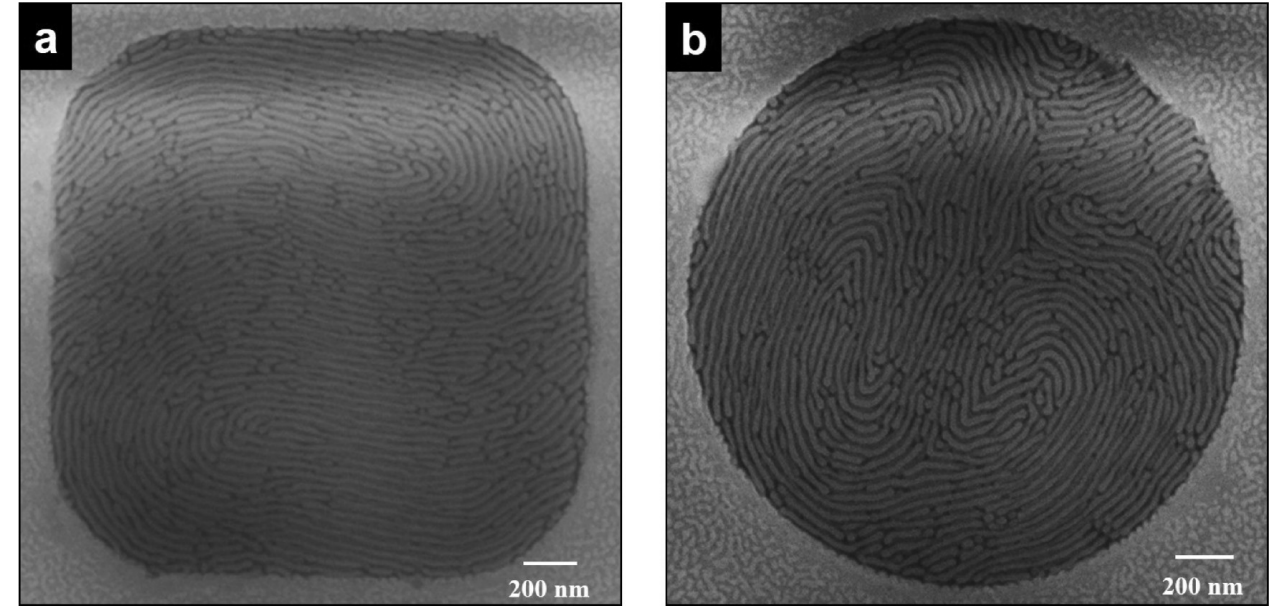

Figure 1. Self-assembly of PS-b-PDMS BCP on NIL resists. (a, b) Top-down SEM images of characteristic PS-b-PDMS "fingerprint" patterns in nanoimprinted circular resists.

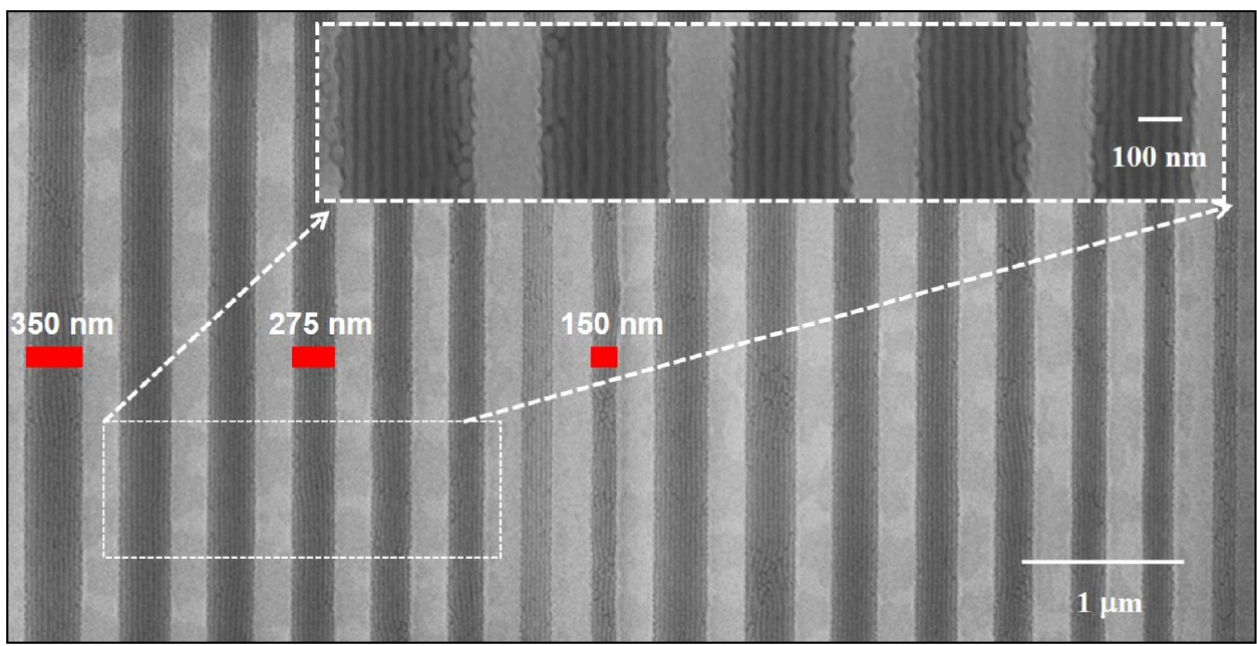

Figure 2. Top-down SEM of PS- $b$-PDMS patterns showing alignment of features over a macroscopic region.

\section{RESULTS AND DISCUSSION}

PS-b-PDMS BCPs have been explored with respect to lithographic patterning for two key reasons: (i) a high FloryHuggins interaction parameter $(\chi \sim 0.26)$ allowing small feature sizes and (ii) high etch contrast stemming from the presence of silicon in the PDMS backbone, allowing a facile pattern transfer to the underlying substrate. Therefore, PS- $b$ PDMS was chosen for this study to produce silicon oxide features that can potentially be used for DSA purposes and to create a demonstration for other technologies requiring precisely located discrete nanofeatures.

Three-dimensional POSS resist topographies were fabricated over large areas using NIL technology (see Experimental Section and Scheme 1). The resin was considered owing to its high modulus that allows high-fidelity nanoimprinting and prevents structure collapse during demolding. ${ }^{27}$ The chemical structure of the resin is presented in Table S1 along with its wetting characteristics. The surface free energies of PS and PDMS are 44.0 and $21.8 \mathrm{mN} / \mathrm{m}^{28}$ respectively. It is evident that the surface free energy of epoxy-POSS is very close to that of PS.

Following the development of POSS geometries, a dilute toluene PS- $b$-PDMS $(31-11 \mathrm{k})$ solution was spin-coated on the POSS guiding patterns. SVA ${ }^{29}$ of the thin PS- $b$-PDMS films in toluene vapors for $3 \mathrm{~h}$ produced microphase-separated features with PDMS cylinders confined within a PS matrix. After SVA, selective etching of films is necessary using $\mathrm{CF}_{4}$ to remove the PDMS polymer/air interface wetting layer and $\mathrm{O}_{2}$ gas to remove the PS matrix and convert PDMS to $\mathrm{SiO}_{2}$. This allows SEM inspection of the remaining oxidized PDMS cylinders (see Scheme 1(iv)).

In our experiments, the initial data presented are based on forming silicon oxide line-space features on conventional architectures, for example, simple flat topographies and parallel topographies to form aligned features as found in DSA works. The SEM images in Figure 1a,b display characteristic silicon oxide line-space features in large POSS topographic areas, such as those developed on planar substrates from PS-b-PDMS BCPs. We have previously shown the self-assembly behavior of PS- $b$-PDMS BCP systems using microwave annealing and SVA on planar and guiding patterns. ${ }^{30-32}$ Additionally, we have illustrated PS- $b$-PDMS alignment on parallel POSS features via SVA. ${ }^{24,33}$ Toluene possesses a solubility parameter of $18.5 \mathrm{M}$ $\mathrm{Pa}^{1 / 2}$ and is a commonly employed solvent for SVA of PS- $b$ PDMS films to induce segregation of the respective blocks. Thus, toluene is primarily selective to the majority PS block. The large scale area shown in Figure $1 \mathrm{~b}$ also exemplifies the superiority of NIL to common optical-based patterning 

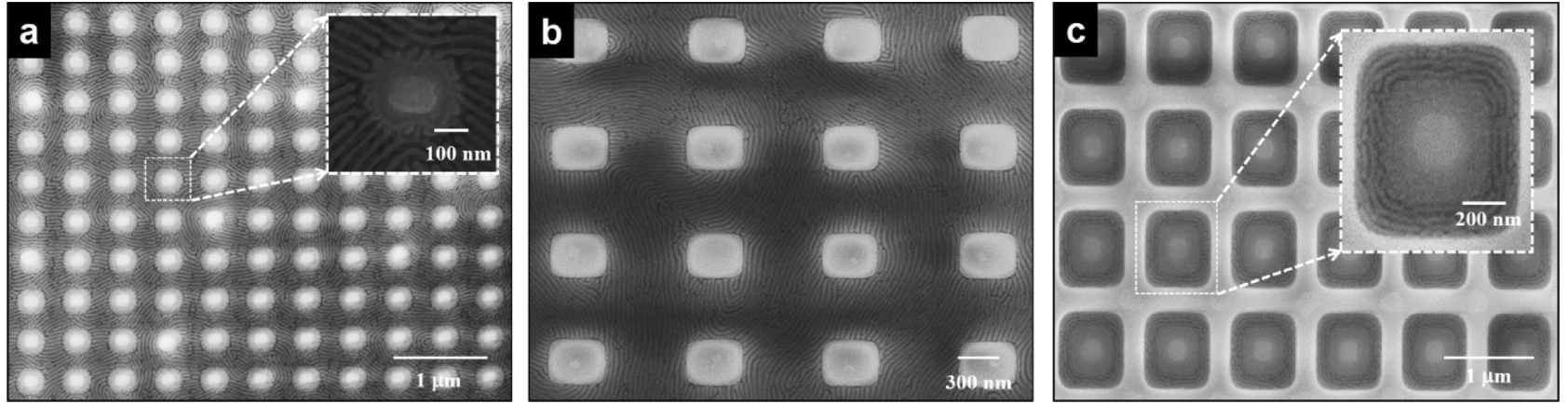

Figure 3. Top-down SEM images of PS- $b$-PDMS line-space features patterned around (a) $\sim 100 \mathrm{~nm}$ POSS postarrays, (b) $\sim 450 \mathrm{~nm}$ POSS postpatterns, and (c) confined within a complex square geometry.
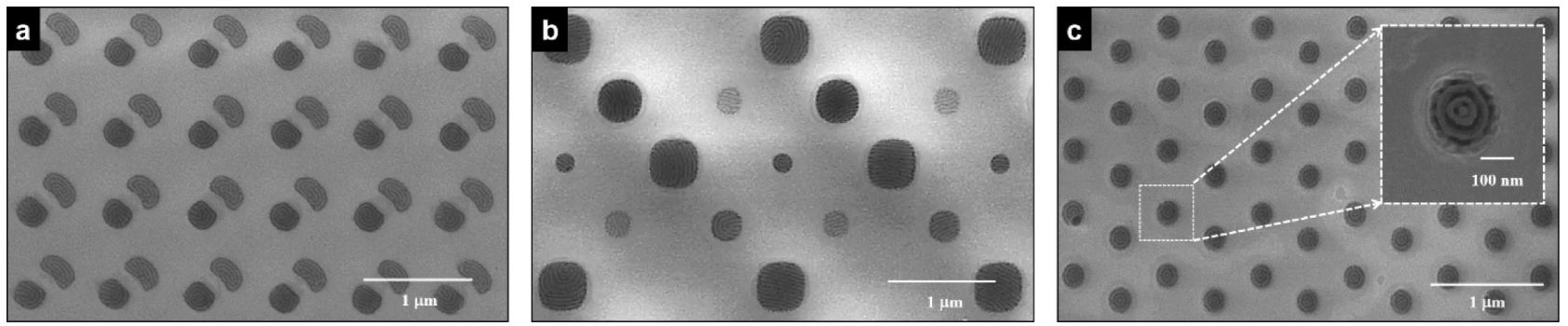

Figure 4. Macroscopic coverage revealed by SEM analysis of PS-b-PDMS patterns showing a circular or ring conformation in (a) irregular patterns, (b) varying circular patterns, and (c) periodically imprinted circular features with equal spacing and size.

methods like EBL, which are restricted because of the long patterning times required. This is a clear demonstration that NIL combined with BCP lithography can allow large scale patterning in a high-throughput fashion. The methods shown here address these two critical requirements for next-generation patterning.

Figure $1 \mathrm{a}, \mathrm{b}$ reveals well-defined silicon oxide line-space features in POSS-generated templates. The feature size and period (i.e., center-to-center spacing) were measured at $\sim 19$ and $\sim 35 \mathrm{~nm}$ (Figure 1a) and at $\sim 18$ and $\sim 36 \mathrm{~nm}$ (Figure 1b).

POSS resists are an extremely effective resist material due to ease of processing for creating fine features and the associated long-term stability for nanolithography use. Equally, the optimization and understanding from our previous studies mentioned above have enabled the tuning of surface-polymer interactions to create defined discrete BCP arrays. In our earlier reports, we presented key results demonstrating that POSS materials can be used with similar efficacy to polymer brushes (e.g., hydroxyl-terminated PS and PDMS) to dictate BCP microdomain orientation to the underlying substrate. ${ }^{24}$ With this knowledge, we have tailored conditions in this work to form uniform silicon oxide arrays in NIL resists.

Figure 2 shows an SEM image of typical DSA approach, where isolated and aligned silicon oxide line-space features can be formed over large areas. One observes that line-space features can be fabricated with good long-range order over macroscopic areas through NIL and BCP lithography. The periodicity of the features is $\sim 35 \mathrm{~nm}$, similar to the dimensions observed on planar topographies in Figure 1. The POSS resist mesas have dimensions of $\sim 245 \mathrm{~nm}$, whereas the channel/ trench widths are varied as indicated in Figure 2. The top-down SEM image shows that varying channel widths can fabricate different numbers of silicon oxide line-space features. For example, trench widths measuring $\sim 150 \mathrm{~nm}$ lead to 5 silicon oxide features, whereas 10 lines can be produced in $350 \mathrm{~nm}$ channel widths, as indicated in the image. This demonstrates the excellent synergistic potential of NIL/BCP techniques to fulfill large scale patterning of features in the deep nanoscale regime. The high-resolution SEM inset in Figure 2 also reveals the uniformity of the silicon oxide features with varying line features with respect to increasing trench size. In contrast, larger trenches do not dictate such strict control of features (see Figure S1, Supporting Information (SI)). The image in Figure 2 shows the ability to alter and manipulate the number of line features within a respective trench. This is a relatively challenging practice with EBL methods over such a small scale area. The inset illustrates the precision that we can tailor an area to include 5 or 10 silicon oxide features depending on the dimensions fabricated by NIL. Such features are ideal for DSA etch mask application, and recent reports have demonstrated high levels of success in pattern transferring silicon oxide masks derived from PS- $b$-PDMS materials to various substrates. ${ }^{34,35}$ Important steps forward have been reported in the literature for pattern transferring PS- $b$-PMDS BCPs leading to high-fidelity features using pulsed laser practices. $^{36}$

The low-resolution SEM image in Figure 3a displays a large scale area $\left(\sim 5 \times 5 \mu \mathrm{m}^{2}\right)$ of silicon oxide line-space features surrounding periodic oval-shaped POSS postfeatures generated through NIL. The image displays retention of the POSS geometry after NIL and characteristic silicon oxide line-space features following patterning with PS- $b$-PDMS and selective plasma-etching procedures (see Experimental Section for details). A uniform monolayer of silicon oxide features is evident with the characteristic BCP fingerprint pattern. Silicon oxide feature size was measured at $\sim 17 \mathrm{~nm}$ with a periodicity $\left(L_{0}\right)$ of $\sim 35 \mathrm{~nm}$. The inset shows that PDMS has an attraction to this circular POSS feature in comparison to the larger features generated in Figure 1a. Figure 3b,c also shows examples of large areas, where oval-shaped POSS posts exist 

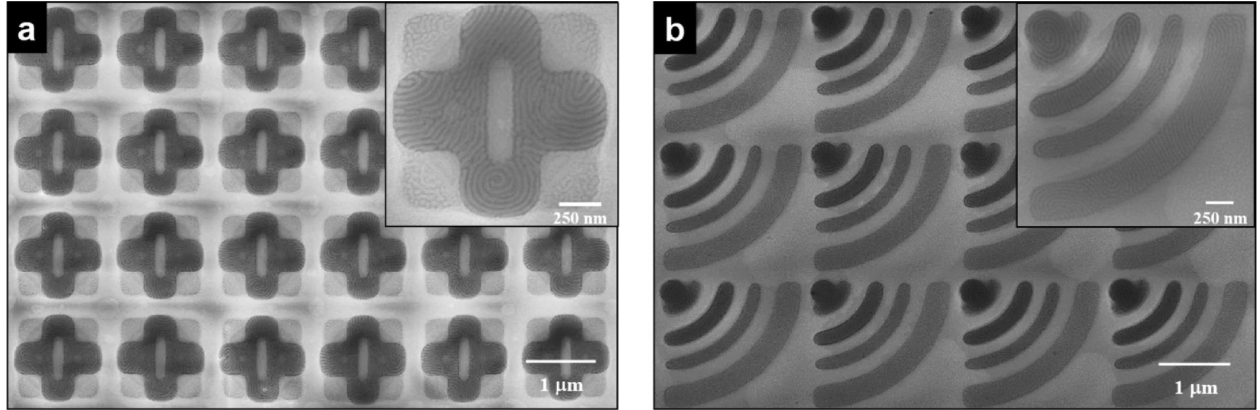

Figure 5. Top-down SEM images of complex imprinted patterns showing regular and uniform PS-b-PDMS patterns in (a) cross-shaped features and (b) banana-like topographies.

combined with silicon oxide features resulting from microphase-separated PS- $b$-PDMS thin films. Figure $3 b$ displays an SEM image of a more "conventional" pattern of line features, whereas Figure $3 c$ shows a more sophisticated pattern geometry driven by the intricate nature of the POSS NIL resist. The square POSS pattern contains a POSS post that fills the centre of the square and is surrounded by aligned selfassembled features. The features shown are unique (see Figure $3 c)$ to previously reported works and further reinforce the ability of BCPs to pattern more complex features than conceived. Furthermore, the types of structures reported here and below add to the capabilities of di-BCP materials to pattern and form exotic structures. This has been made possible due to the excellent microphase separation ability of PS- $b$-PDMS. Moreover, NIL has the ability to fabricate uniform topographic resists over large areas (i.e., macroscale) with intricate patterns surpassing those produced by EBL or other slow and costly lithographic methods.

Figure $4 a-c$ displays a range of intriguing POSS topographies following BCP patterning with PS- $b$-PDMS. Figure $4 \mathrm{a}$ is composed of two varying circular guiding topographies, where silicon oxide features assemble in a concentric manner. One should note the excellent uniform curvature of the silicon oxide nanostructures within the POSS resists in Figure $4 a-c$. This is not only a result of the high interfacial width between PS and PDMS (due to the material's high $\chi$ ) but also due to the nature of the POSS sidewalls. The SEM image in Figure 4a shows the excellent performance potential of NIL technology with a "high- $\chi$ " BCP material to pattern concentric features. In contrast, the wider, less-defined topographic patterns displayed in Figure $4 \mathrm{~b}$ did not result in concentric self-assembly of the PS- $b$-PDMS BCP. Fingerprint patterns were observed in the larger topographic patterns and in the smaller-diameter topographic patterns. With more uniform periodic cylindershaped topographies, concentric patterns with high reproducibility across large areas resulted, as shown in Figure 4c. The high-resolution SEM inset in Figure $4 c$ also shows that the PDMS dominates the inner part of the pattern. This has been reported to occur as a result of the diameter of the topography, as was previously outlined for PS- $b$-PMMA BCP systems. ${ }^{17}$ These uniform structures could be used as templates for (i) pattern-transfer masks to create diverse silica nanostructures or (ii) incorporating metal oxide materials via evaporation or sputtering methods. More intricate examples of 3-D POSS geometries with PS- $b$-PDMS features are displayed in Figures S2 and S3, SI.

Figure 5a displays silicon oxide line features after selfassembly and etching on a cross-imprinted POSS pattern with the presence of a POSS line at the center. One observes characteristic fingerprint structures in the open area of the topographic template. This is evident from the inset in Figure 5a, where nanofeatures with no directed assembly structures are formed. However, such features demonstrating large-scale control of uniform assembly and location (i.e., within NIL topographies) may be useful in bioassay studies, where sensitivity could be analyzed in patterned versus unpatterned areas. More complex patterns have also been explored as shown in Figure 5b. Self-assembly was achieved in "banana"-like topographies that are of varying widths and lengths. Welldefined uniform silicon oxide features are generated, as seen in the inset in Figure $5 b$.

\section{CONCLUSIONS}

The results presented and discussed in this article bring forward the areas of BCP patterning and NIL technology for unconventional topographic geometries. The synergistic approach of confining PS- $b$-PDMS thin films within an NIL POSS resist produced excellent silicon oxide nanofeatures for both DSA application and more exotic 3-D topographic resist patterns. More work is required to achieve better alignment in the nontraditional templates used here so that the overall process can be applied in nanoapplications. The patterned features also contribute to the area of "soft" graphoepitaxy using a light-sensitive material that can be patterned with high uniformity over macroscopic areas, as demonstrated here.

\section{ASSOCIATED CONTENT}

\section{S Supporting Information}

The Supporting Information is available free of charge on the ACS Publications website at DOI: 10.1021/acsomega.7b00781.

Epoxy-POSS structure and wetting properties (Table S1); PS- $b$-PDMS pattern formation in different geometries (Figures S1-S3) (PDF)

\section{AUTHOR INFORMATION}

\section{Corresponding Author}

*E-mail: morrism2@tcd.ie. Tel: +353(0) 18963089.

\section{Notes}

The authors declare no competing financial interest.

\section{ACKNOWLEDGMENTS}

The authors gratefully acknowledge financial support for this work provided by the EU FP7 NMP project, LAMAND Project (Grant Number 245565), and the Science Foundation Ireland (Grant Number 12/RC/2278). C.S., A.F., N.K., and C.M.S.-T. 
acknowledge support from the MINECO S Ochoa grant SEV2013-0295 and the project PHENTOM (FIS2015-70862-P). The authors thank the staff of the Advanced Microscopy Laboratory (AML, Trinity College, Dublin) for characterization assistance.

\section{REFERENCES}

(1) Biswas, A.; Bayer, I. S.; Biris, A. S.; Wang, T.; Dervishi, E.; Faupel, F. Advances in top-down and bottom-up surface nanofabrication: Techniques, applications \& future prospects. Adv. Colloid Interface Sci. 2012, 170, 2-27.

(2) Hobbs, R. G.; Petkov, N.; Holmes, J. D. Semiconductor nanowire fabrication by bottom-up and top-down paradigms. Chem. Mater. 2012, 24, 1975-1991.

(3) Xiao, S.; Yang, X.; Hwu, J. J.; Lee, K. Y.; Kuo, D. A facile route to regular and nonregular dot arrays by integrating nanoimprint lithography with sphere-forming block copolymer directed selfassembly. J. Polym. Sci., Part B: Polym. Phys. 2014, 52, 361-367.

(4) Schift, H. Nanoimprint lithography: An old story in modern times? A review. J. Vac. Sci. Technol., B: Microelectron. Nanometer Struct.-Process., Meas., Phenom. 2008, 26, 458-480.

(5) Guo, L. J. Nanoimprint Lithography: Methods and Material Requirements. Adv. Mater. 2007, 19, 495-513.

(6) Jeong, S.-J.; Kim, J. Y.; Kim, B. H.; Moon, H.-S.; Kim, S. O. Directed self-assembly of block copolymers for next generation nanolithography. Mater. Today 2013, 16, 468-476.

(7) Morris, M. A. Directed self-assembly of block copolymers for nanocircuitry fabrication. Microelectron. Eng. 2015, 132, 207-217.

(8) Ross, C. A.; Berggren, K. K.; Cheng, J. Y.; Jung, Y. S.; Chang, J.-B. Three-Dimensional Nanofabrication by Block Copolymer SelfAssembly. Adv. Mater. 2014, 26, 4386-4396.

(9) Cummins, C.; Ghoshal, T.; Holmes, J. D.; Morris, M. A. Strategies for Inorganic Incorporation using Neat Block Copolymer Thin Films for Etch Mask Function and Nanotechnological Application. Adv. Mater. 2016, 28, 5586-5618.

(10) Xiao, S.; Yang, X.; Edwards, E. W.; La, Y.-H.; Nealey, P. F. Graphoepitaxy of cylinder-forming block copolymers for use as templates to pattern magnetic metal dot arrays. Nanotechnology 2005, 16, S324-S329.

(11) Tsai, H.; Pitera, J. W.; Miyazoe, H.; Bangsaruntip, S.; Engelmann, S. U.; Liu, C.-C.; Cheng, J. Y.; Bucchignano, J. J.; Klaus, D. P.; Joseph, E. A.; Sanders, D. P.; Colburn, M. E.; Guillorn, M. A. Two-Dimensional Pattern Formation Using Graphoepitaxy of PS- $b$ PMMA Block Copolymers for Advanced FinFET Device and Circuit Fabrication. ACS Nano 2014, 8, 5227-5232.

(12) Farrell, R. A.; Kinahan, N. T.; Hansel, S.; Stuen, K. O.; Petkov, N.; Shaw, M. T.; West, L. E.; Djara, V.; Dunne, R. J.; Varona, O. G.; Gleeson, P. G.; Jung, S.-J.; Kim, H.-Y.; Koleśnik, M. M.; Lutz, T.; Murray, C. P.; Holmes, J. D.; Nealey, P. F.; Duesberg, G. S.; Krstić, V.; Morris, M. A. Large-scale parallel arrays of silicon nanowires via block copolymer directed self-assembly. Nanoscale 2012, 4, 3228-3236.

(13) Borah, D.; Rasappa, S.; Senthamaraikannan, R.; Holmes, J. D.; Morris, M. A. Graphoepitaxial Directed Self-Assembly of PolystyreneBlock-Polydimethylsiloxane Block Copolymer on Substrates Functionalized with Hexamethyldisilazane to Fabricate Nanoscale Silicon Patterns. Adv. Mater. Interfaces 2014, 1, No. 1300102.

(14) Borah, D.; Rasappa, S.; Shaw, M. T.; Hobbs, R. G.; Petkov, N.; Schmidt, M.; Holmes, J. D.; Morris, M. A. Directed self-assembly of PS-b-PMMA block copolymer using HSQ lines for translational alignment. J. Mater. Chem. C 2013, 1, 1192-1196.

(15) Cummins, C.; Gangnaik, A.; Kelly, R. A.; Borah, D.; O’Connell, J.; Petkov, N.; Georgiev, Y. M.; Holmes, J. D.; Morris, M. A. Aligned silicon nanofins via the directed self-assembly of PS- $b$-P4VP block copolymer and metal oxide enhanced pattern transfer. Nanoscale 2015, 7, 6712-6721.

(16) Cummins, C.; Gangnaik, A.; Kelly, R. A.; Hydes, A. J.; O'Connell, J.; Petkov, N.; Georgiev, Y. M.; Borah, D.; Holmes, J. D.; Morris, M. A. Parallel Arrays of Sub-10 nm Aligned Germanium
Nanofins from an In Situ Metal Oxide Hardmask using Directed SelfAssembly of Block Copolymers. Chem. Mater. 2015, 27, 6091-6096.

(17) Jeong, S.-J.; Kim, J. E.; Moon, H.-S.; Kim, B. H.; Kim, S. M.; Kim, J. B.; Kim, S. O. Soft Graphoepitaxy of Block Copolymer Assembly with Disposable Photoresist Confinement. Nano Lett. 2009, 9, 2300-2305.

(18) Kim, S. O.; Solak, H. H.; Stoykovich, M. P.; Ferrier, N. J.; de Pablo, J. J.; Nealey, P. F. Epitaxial self-assembly of block copolymers on lithographically defined nanopatterned substrates. Nature 2003, 424, 411-414.

(19) Liu, C.-C.; Ramírez-Hernández, A.; Han, E.; Craig, G. S. W.; Tada, Y.; Yoshida, H.; Kang, H.; Ji, S.; Gopalan, P.; de Pablo, J. J.; Nealey, P. F. Chemical Patterns for Directed Self-Assembly of Lamellae-Forming Block Copolymers with Density Multiplication of Features. Macromolecules 2013, 46, 1415-1424.

(20) Onses, M. S.; Song, C.; Williamson, L.; Sutanto, E.; Ferreira, P. M.; Alleyne, A. G.; Nealey, P. F.; Ahn, H.; Rogers, J. A. Hierarchical patterns of three-dimensional block-copolymer films formed by electrohydrodynamic jet printing and self-assembly. Nat. Nanotechnol. 2013, 8, 667-675.

(21) Park, S.-M.; Liang, X.; Harteneck, B. D.; Pick, T. E.; Hiroshiba, N.; Wu, Y.; Helms, B. A.; Olynick, D. L. Sub-10 nm Nanofabrication via Nanoimprint Directed Self-Assembly of Block Copolymers. ACS Nano 2011, 5, 8523-8531.

(22) Xiao, S.; Yang, X.; Steiner, P.; Hsu, Y.; Lee, K.; Wago, K.; Kuo, D. Servo-Integrated Patterned Media by Hybrid Directed SelfAssembly. ACS Nano 2014, 8, 11854-11859.

(23) Yang, X.; Xiao, S.; Hu, W.; Hwu, J.; van de Veerdonk, R.; Wago, K.; Lee, K.; Kuo, D. Integration of nanoimprint lithography with block copolymer directed self-assembly for fabrication of a sub- $20 \mathrm{~nm}$ template for bit-patterned media. Nanotechnology 2014, 25, No. 395301.

(24) Borah, D.; Rasappa, S.; Salaun, M.; Zellsman, M.; Lorret, O.; Liontos, G.; Ntetsikas, K.; Avgeropoulos, A.; Morris, M. A. Soft Graphoepitaxy for Large Area Directed Self-Assembly of Polystyreneblock-Poly(dimethylsiloxane) Block Copolymer on Nanopatterned POSS Substrates Fabricated by Nanoimprint Lithography. Adv. Funct. Mater. 2015, 25, 3425-3432.

(25) Jung, Y. S.; Ross, C. A. Orientation-Controlled Self-Assembled Nanolithography Using a Polystyrene-Polydimethylsiloxane Block Copolymer. Nano Lett. 2007, 7, 2046-2050.

(26) Żenkiewicz, M. Comparative study on the surface free energy of a solid calculated by different methods. Polym. Test. 2007, 26, 14-19.

(27) Pina-Hernandez, C.; Guo, L. J.; Fu, P.-F. High-Resolution Functional Epoxysilsesquioxane-Based Patterning Layers for LargeArea Nanoimprinting. ACS Nano 2010, 4, 4776-4784.

(28) Drummond, C. J.; Chan, D. Y. C. van der Waals Interaction, Surface Free Energies, and Contact Angles: Dispersive Polymers and Liquids. Langmuir 1997, 13, 3890-3895.

(29) Sinturel, C.; Vayer, M.; Morris, M.; Hillmyer, M. A. Solvent Vapor Annealing of Block Polymer Thin Films. Macromolecules 2013, 46, 5399-5415.

(30) Borah, D.; Senthamaraikannan, R.; Rasappa, S.; Kosmala, B.; Holmes, J. D.; Morris, M. A. Swift Nanopattern Formation of PS-bPMMA and PS- $b$-PDMS Block Copolymer Films Using a Microwave Assisted Technique. ACS Nano 2013, 7, 6583-6596.

(31) Borah, D.; Shaw, M. T.; Holmes, J. D.; Morris, M. A. Sub-10 nm Feature Size PS- $b$-PDMS Block Copolymer Structures Fabricated by a Microwave-Assisted Solvothermal Process. ACS Appl. Mater. Interfaces 2013, 5, 2004-2012.

(32) Borah, D.; Rasappa, S.; Senthamaraikannan, R.; Holmes, J. D.; Morris, M. A. Tuning PDMS Brush Chemistry by $\mathrm{UV}-\mathrm{O}_{3}$ Exposure for PS-b-PDMS Microphase Separation and Directed Self-assembly. Langmuir 2013, 29, 8959-8968.

(33) Borah, D.; Simao, C. D.; Senthamaraikannan, R.; Rasappa, S.; Francone, A.; Lorret, O.; Salaun, M.; Kosmala, B.; Kehagias, N.; Zelsmann, M.; Sotomayor-Torres, C. M.; Morris, M. A. Softgraphoepitaxy using nanoimprinted polyhedral oligomeric silsesquiox- 
ane substrates for the directed self-assembly of PS- $b$-PDMS. Eur. Polym. J. 2013, 49, 3512-3521.

(34) Li, T.; Wang, Z.; Schulte, L.; Hansen, O.; Ndoni, S. Fast \& scalable pattern transfer via block copolymer nanolithography. RSC Adv. 2015, 5, 102619-102624.

(35) Li, T.; Wang, Z.; Schulte, L.; Ndoni, S. Substrate tolerant direct block copolymer nanolithography. Nanoscale 2016, 8, 136-140.

(36) Girardot, C.; Böhme, S.; Archambault, S.; Salaün, M.; LatuRomain, E.; Cunge, G.; Joubert, O.; Zelsmann, M. Pulsed Transfer Etching of PS-PDMS Block Copolymers Self-Assembled in $193 \mathrm{~nm}$ Lithography Stacks. ACS Appl. Mater. Interfaces 2014, 6, 1627616282. 